\title{
Modeling of fiber-optic sensors based on micromechanical vibrations in liquid
}

\author{
A. M. Prokhorov, R. O. Claus, A. L. Popov, and T. V. Tulaikova
}

Fiber-optic chemical sensors based on optical power absorption or wavelength changes are well known. A new type of sensing element is considered. A micromechanical vibrated fiber-optic tip changes its resonance frequency during its operation. Sensors of this type are simple and convenient and do not require adjustment while in use. They are useful in industry and in medical applications. The action of this sensitive element in a liquid is considered. (C) 1997 Optical Society of America

Key words: Fiber, chemical sensor, vibrations, string.

\section{Principle of Operation}

Modulated optical power is launched into the fiber from a laser or a light-emitting diode (Fig. 1). The bending oscillations of the fiber tip ${ }^{1}$ (or silicon string ${ }^{2}$ ) have a known resonance frequency determined by the size and the mass of the tip. During chemical sensor operation, the molecules from the surrounding liquid mixture are absorbed by the cladding of the vibrated tip, thus changing its mass $m$. The resulting change in resonant frequency is dependent on the number of absorbed detected molecules. By monitoring this change, we can analyze the concentration of the molecules in a liquid mixture. This sensor can also be applied to gases.

\section{Analytical Consideration}

Theoretical analysis was performed for vibrations of the quartz cantilever with a circular cross section with a thin coating of absorbed molecules. For the sensor operation, we must consider the influence of the additional mass for a dense medium, that is, for a surrounding liquid. In this case we present a mathematical description of the problem of joint vibration of the sensor and the additional mass of the liquid.

A. M. Prokhorov is with the General Physics Institute, Russian Academy of Sciences, 38 Vavilova Street, Moscow, Russia. R. O. Claus is with the Fiber and Electro-Optics Research Center, Virginia Polytechnic Institute and State University, Blacksburg, Virginia 24061. A. L. Popov and T. V. Tulaikova are with the Institute for Problems in Mechanics, Russian Academy of Sciences, 101 Prospect Vernadskogo, 117526 Moscow, Russia.

Received 16 October 1996; revised manuscript received 21 January 1997.

0003-6935/97/225562-04\$10.00/0

(C) 1997 Optical Society of America
This consideration includes the equation of transverse cantilever oscillation [Eq. (1) $]^{3}$ with the additional term for the liquid response. The liquid action is expressed as an additional pressure $P_{S}$ on the sensor surface $S$ :

$$
E J \frac{\partial^{4} y}{\partial x^{4}}+\rho F \frac{\partial^{2} y}{\partial t^{2}}+\left.2 \pi r_{0} P\right|_{s}=0
$$

where $\rho$ and $F$ are the fiber material densities and the cross-section squares, $F=\pi r_{0}{ }^{2}, E$ is the cantilever elasticity module, $y(x, t)$ is a transverse displacement of the fiber cantilever, $t$ is time, $P$ is pressure in a liquid, and $S$ is a cantilever surface. Also, $2 \pi r_{0} P$ is an amount of the liquid for a cantilever and $J=$ $\pi r_{0}{ }^{4} / 4$ is the second rotation moment. The pressure must conform to the wave equation for the liquid around the fiber tip. The wave equation must be expressed by the cylindrical coordinate system appropriate to the cylindrical fiber cantilever. There $r$ and $\varphi$ represent the radius and the angle, respectively, of the cylindrical coordinate system with the center in the fiber core axis, and $x$ is the direction that coincides with the fiber axis.

$$
\frac{\partial^{2} P}{\partial r^{2}}+\frac{1}{r} \frac{\partial P}{\partial r}+\frac{\partial^{2} P}{\partial x^{2}}+\frac{1}{r^{2}} \frac{\partial^{2} P}{\partial \varphi^{2}}-\frac{1}{C_{0}^{2}} \frac{\partial^{2} P}{\partial t^{2}}=0,
$$

where $C_{0}$ is the velocity of sound in a liquid.

The appropriate boundary condition is the nonflow equation on the cantilever surface:

$$
\left.\frac{\partial P}{\partial r}\right|_{r=r_{0}}=-\rho_{0} \frac{\partial^{2} y}{\partial t^{2}},
$$




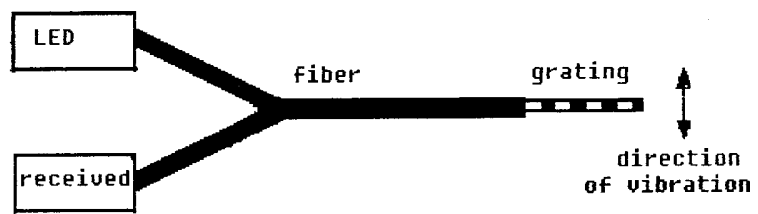

Fig. 1. Scheme of the sensor.

where $\rho_{0}$ is the liquid density. Last, the fading condition for the excess pressure away from the cantilever is

$$
\frac{\partial P}{\partial r}+i k P=O\left(\frac{1}{r}\right), r \rightarrow \infty .
$$

The solution of Eqs. (1)-(4) for joint oscillation of the fiber cantilever and a liquid has the harmonic function:

$$
\begin{aligned}
y(x, t) & =W(x) \exp (-i \omega t), \\
P(r, x, \varphi, t) & =P(r, x) \exp [i(\varphi-\omega t)],
\end{aligned}
$$

where $\omega=2 \pi f$ is the angular frequency.

Substituting Eq. (5) in Eqs. (1)-(3), we obtain the system of the main equations in the form

$$
\begin{aligned}
E J W^{\mathrm{IV}}(x)+\rho F \omega^{2} W(x)+2 \pi r P(x, r) & =0, \\
(0<x<1, r & \left.=r_{0}\right), \\
\frac{\partial^{2} P}{\partial r^{2}}+\frac{1}{r} \frac{\partial P}{\partial r}+\frac{\partial^{2} P}{\partial x^{2}}+\left(k^{2}-\frac{1}{r^{2}}\right) P & =0, \\
k=\frac{\omega}{C_{0}},\left.\frac{\partial P}{\partial r}\right|_{r=r_{0}} & =\omega^{2} \rho_{0} W(x) .
\end{aligned}
$$

We can make an assumption about the decrease in the value of pressure $P(r, x)$ far from the fiber cantilever surface. $P(r, x)$ is assumed in the more convenient form:

$$
P(r, x)=P\left(r_{0}, x\right) \exp \left[a\left(r_{0}-r\right)\right], \operatorname{Re}(\mathrm{a})>0 .
$$

In Eq. (7) $a$ is the parameter that must be determined, so we calculate from Eq. (6)

$$
P\left(x, r_{0}\right)=-\frac{\omega^{2} \rho_{0}}{a} W(x) .
$$

The remaining equations from the initial system for the cantilever in accordance with Eq. (8) provide the following system:

$$
\begin{gathered}
E J W^{\mathrm{IV}}(x)-\omega^{2}\left(\rho F+\frac{2 \pi \rho_{0} r_{0}}{a}\right) W(x)=0, \\
W^{\mathrm{II}}(x)+\left(k^{2}-\frac{1}{r_{0}{ }^{2}}+a^{2}-\frac{a}{r_{0}}\right) W(x)=0 .
\end{gathered}
$$

The dispersion equation for the gas medium was obtained for the fiber cantilever ${ }^{1}$ without consideration of the liquid influence. It has a convenient form:

$$
\cos (\alpha l) \cosh (\alpha l)=-1,
$$

where, for different modes of vibrations, $l \alpha_{1}=1.875$, $l \alpha_{2}=4.694$, and $l \alpha_{3}=7.85$ for $l=1 \mathrm{~mm}$, but $l \alpha_{k}=$ $0.5 \pi(2 k-1)$ for $k=4,5,6$.

But this dispersion equation in a liquid medium has the form

$$
E J\left(a^{2}-\frac{a}{r_{0}}+k^{2}-\frac{1}{r_{0}^{2}}\right)^{2}-\omega^{2} \rho F\left(1+2 \frac{\rho_{0}}{a r_{0} \rho}\right)=0 .
$$

This is the algebraic equation for the $a$ determination. By substituting $\chi=a r_{0} \gg 1$, we can get the simpler equation:

$$
\chi^{5}-4 \lambda^{2}\left(\chi+\frac{2 \rho_{0}}{\rho}\right)=0, \lambda=\omega r_{0} / C, C=\left(\frac{E}{\rho}\right)^{1 / 2} .
$$

As this equation can be solved numerically, it can be proved that one of the roots, $\chi_{1}$, has a positive sign. The equation shows the frequency reduction resulting from the density of the medium. For a surrounding medium (liquid) that is dense the root of Eq. (12) can be calculated approximately as

$$
\chi_{1}=\left(6 \frac{\lambda^{2} \rho_{0}}{\rho}\right)^{1 / 5} .
$$

For gases, the first root is

$$
\chi_{1}=(4 \lambda s 2)^{1 / 4}=(2 \lambda)^{1 / 2} .
$$

In the main equation for vibrations, the additional mass of the liquid is considered by the additional last term with the value of $\chi_{1}$ from Eq. (13):

$$
E J W^{\mathrm{IV}}(x)-\omega^{2} \rho F\left(1+\frac{2 \rho_{0}}{\chi_{1} \rho}\right) W=0 .
$$

In addition to Eq. (10), the relation of the $\alpha$ and the $\omega$ as described in Ref. 2 is necessary. There it has the form

$$
\alpha^{4}=\omega^{2} \frac{\rho F}{E J}\left(1+\frac{2 \rho_{0}}{\chi_{1} \rho}\right),
$$

and $\chi_{1}$ must be calculated from Eq. (13) or Eq. (14) for liquid or gas. One can find easily the coupling of the $J$ and $F$ values in the form

$$
\left(\frac{J}{F}\right)^{1 / 2}=\frac{r}{2} .
$$

We have considered here the resonance frequency of vibrations of the fiber-optic cantilever. The frequency as a function of its length $l$ and its fiber radius $r$ is presented in Figs. 2-4. One can see that the resonance frequencies increase with an increase in the fiber radius (Fig. 2) according to Eqs. (16) and (16a) and decrease strongly with the tip-length increase (Fig. 4). For high sensitivity for the sensor fiber tip it is best if sizes are long in length and small in diameter because changes in frequency are large during chemical sensor operation because of an increase in the fiber diameter during absorption of the molecules. In practice a fiber-tip diameter of $1 \mathrm{~mm}$ 


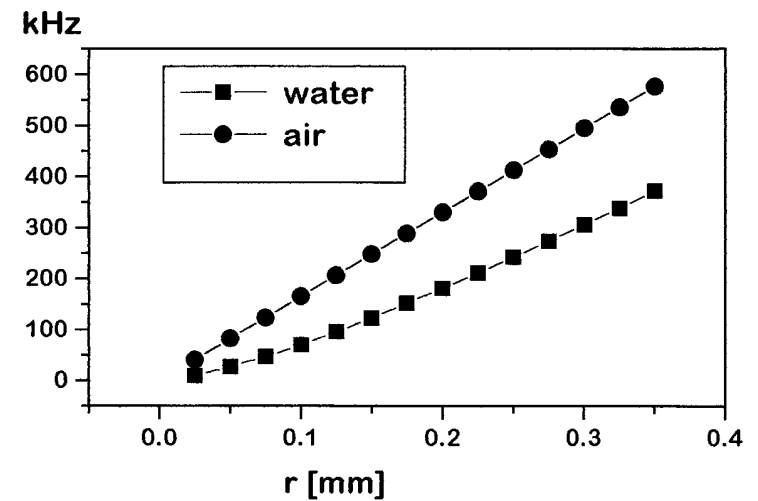

Fig. 2. Resonance frequency as a function of fiber radius; fiber length, $1 \mathrm{~mm}$.

is probably more convenient (Fig. 3). In Fig. 1 of our previous paper ${ }^{1}$ we pointed to the Bragg-like diffraction grating spectrum change to fix the fiber-tip cantilever deviations.

With these results the resonant frequency can be determined for a vibrated fiber-optic sensor tip in a liquid. We reduced the sensor frequency $\omega_{0}$ by placing this tip in water, and we achieved a value of $\omega_{0} / \omega$ $=4$.

During chemical sensor operation the detected molecules are absorbed into the fiber-tip surface as

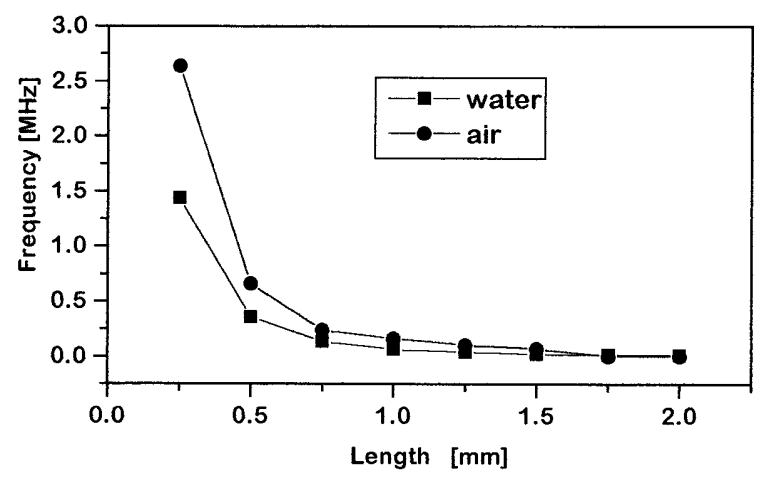

Fig. 3. Resonance frequency as fiber length increases; fiber radius, $0.1 \mathrm{~mm}$.

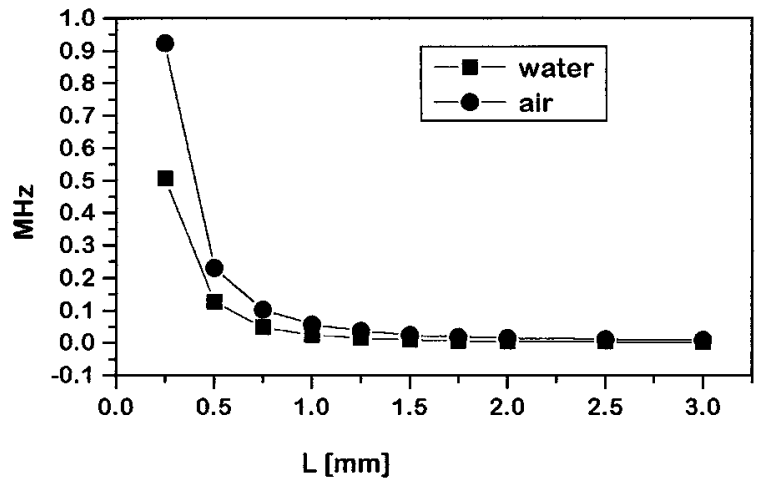

Fig. 4. Resonance frequency as fiber length increases; fiber radius, $0.035 \mathrm{~mm}$. its cladding, with a thickness $h$ and material density $\rho_{*}$. With the fiber cladding increase during the absorption of detected molecules, we can write the main equation of vibrations [Eq. (9)] with an additional final term:

$$
E J W^{\mathrm{IV}}(x)-\omega^{2}\left(\rho F+\frac{2 \pi \rho_{0} r_{0}}{a}+\rho F_{*}\right) W(x)=0,
$$

where $\rho_{*}, F_{*}$ are the density and the cross section of the layer of detected molecules at the chemical sensor tip during its work. If

$$
F_{*} \approx 2 \pi h r
$$

Eq. (11) will change to

$E J\left(a^{2}-\frac{a}{r_{0}}+k^{2}-\frac{1}{r_{0}^{2}}\right)^{2}-\omega^{2} \rho F\left(1+\frac{2 \rho_{0}}{a r_{0} \rho}+\frac{\rho_{*} F_{*}}{\rho F}\right)=0$.

With the additional cladding that was formed from the absorbed molecules, the dispersion equation [see Eq. (12)] takes the form

$$
\chi^{5}-4 \lambda^{2}\left[\chi\left(1+\frac{\rho_{*} F_{*}}{\rho F}\right)+2 \frac{\rho_{0}}{\rho}\right]=0 .
$$

The equation for the relation $\alpha$ and $\omega$ with absorbedmolecule cladding is transformed from that of Eq. (16) to

$$
\alpha^{4}=\omega^{2} \frac{\rho F}{E J}\left(1+\frac{\rho_{*} F_{*}}{\rho F}+\frac{2 \rho_{0}}{\chi_{1} \rho}\right) .
$$

To calculate the resonance frequency during a sensor operation in a denser medium, we must first obtain $\alpha$ with Eq. (10), and calculate Eq. (16) with Eq. (13) or Eq. (14) to find $\chi_{1}$ and $\omega$.

The result of the joint calculation of Eqs. (10), (20), and (21) permits us to find the relation of the fibertip-resonance frequency and the thickness of the absorbed detected molecules (Fig. 5).

We take the following dimensions for further consideration: $l=0.3 \mathrm{~mm}, r=0.035 \mathrm{~mm}$. For practical applications, for medicine in particular, this is a

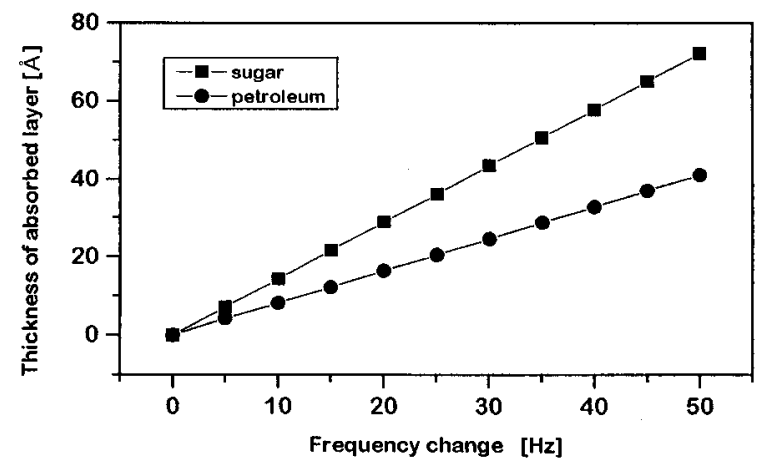

Fig. 5. Sensor operation in sugar solution and in petroleum solution. 
small and a convenient sensor. It can measure the changes of the admixture concentration during chemical or clinical analysis. Also this sensor can be useful as a simple instrument for density measurements in a liquid or for pressure measurements of gases. The derivations of the $\omega$ to $l$ and to $r$ gives the strong dependence of the frequency as $l^{-3}$.

Finally, Fig. 5 demonstrates the process of frequency change during molecule absorption for two interesting practical cases. The upper curve corresponds to sugar absorption (density $\rho_{*}=1.525 \mathrm{~g} /$ $\mathrm{sm}^{3}$ ) in a medical application of this sensor. The lower curve corresponds to petroleum pollution $\left(\rho_{*}=\right.$ $0.866 \mathrm{~g} / \mathrm{sm}^{3}$ ) in water. This sensor can detect easily the absorbed molecular thickness of $1 \AA$.

\section{Conclusions}

In this research a new type of sensitive sensor element is considered: the fiber-optic micromechanical vibrated tip was investigated and optimized. Equations for the fiber cantilever resonance-frequency calculations in a gas and in water were achieved. The original method was developed for the joint solution of the vibrations of a fiber cantilever and a surrounding liquid.

\section{References}

1. A. L. Popov and T. V. Tulaikova, "Chemical sensor using fiber optical micromechanical vibrations," in Digest of Technical Papers, Eighth International Conference on Solid-State Sensors and Activators, Transducers-95 Eurosensors IX (Norstedts, Stockholm, 1995), pp. 437-440.

2. L. M. Zhang, D. Uttamchandani, and B. Culshaw, "Measurements of the mechanical properties of silica microresonators," Sensors Actuators A 29, 79-84 (1991).

3. M. C. Junger and D. Feit, Sound, Structures and Their Interaction (MIT Press, Cambridge, Mass., 1972), p. 470. 